\title{
Alopecia totalis and vitiligo in common variable immunodeficiency
}

\author{
G. Spickett ${ }^{1}$, A.G. Prentice ${ }^{2}$ T. Wallington ${ }^{3}$, A.D.B. Webster ${ }^{4}$ and H. Chapel ${ }^{1}$ \\ ${ }^{1}$ Department of Immunology, John Radcliffe Hospital, Oxford, ${ }^{2}$ Derriford Hospital, Plymouth, ${ }^{3}$ South \\ Western Regional Transfusion Centre, Bristol, ${ }^{4}$ Clinical Research Centre, Northwick Park Hospital, \\ Harrow, UK
}

\begin{abstract}
Summary: Three cases of severe and irreversible alopecia occurring in patients with common variable immunodeficiency are described. In all three cases, hair loss developed after the diagnosis of immune deficiency; one of the patients also had extensive vitiligo. A fourth patient had vitiligo in the absence of alopecia. No change in the alopecia or vitiligo was noted in any patient as a result of immunoglobulin replacement therapy.
\end{abstract}

\section{Introduction}

Common variable immunodeficiency (CVID) is a disorder characterized by a failure of functional antibody production, leading to recurrent bacterial infections. ${ }^{1}$ In the UK there are an estimated $800-1000$ patients, although the number may be much higher as accurate figures are not yet available. Onset may be at any age, although there are peaks of incidence in early childhood and early adulthood. The cause is unknown. The usual presentation is with recurrent sinopulmonary infections. In $20 \%$ of patients, autoimmune phenomena occur, including haemolytic anaemia, thrombocytopenia, neutropenia and thyroid disease. ${ }^{2}$ Autoantibodies may occur but are difficult to detect. Gastritis also occurs but is not associated with anti-parietal cell antibodies. Vitiligo and alopecia areata have been reported in this condition: Ipp \& Gelfand report three hypogammaglobulinaemic patients, one of whom had the congenital (X-linked) form, with alopecia totalis. ${ }^{3}$ Asherson \& Webster have reported three cases of vitiligo in CVID, and one patient with CVID and alopecia. ' Garcia et al. have reported a single case of alopecia areata, associated with CVID,${ }^{4}$ and Tan \& Samman have described alopecia and lichen planus occurring in a patient with thymoma and hypogammaglobulinaemia. ${ }^{5}$

We now report three cases of CVID accompanied by alopecia, in one case also by vitiligo, and one case of vitiligo alone. We discuss the aetiology of these conditions in the light of their occurrence in immunodeficient patients.

\section{Case reports}

Case 1

A man, born in 1959, first presented aged 4 , when he underwent a tonsillectomy. Following this, he was troubled by recurrent 'sterile' knee effusions, and joint stiffness throughout childhood. He had infectious hepatitis in 1969, and in 1970 diabetes mellitus was diagnosed and he was commenced on insulin. Recurrent sinopulmonary infections and steatorrhoea began at this time, and during investigation in 1978, hypogammaglobulinaemia was noted. Replacement therapy was not started until 1983, when he contracted a serious salmonella infection in Portugal, followed by a perirectal abscess, which required surgical drainage. In the same year, alopecia was noted and progressed to total hair loss. His mean cell volume (MCV) was elevated and a diagnosis of pernicious anaemia made with autoantibodies only to intrinsic factor. Replacement vitamin B12 therapy was begun. He was then given intravenous immunoglobulin [IVIg] therapy, $12 \mathrm{~g} /$ three weekly, and more recently, $25 \mathrm{~g} / \mathrm{two}$ weekly. During IVIg therapy there has been no effect on hair growth. He still occasionally has low serum levels of calcium, magnesium, zinc and iron; his diarrhoea fluctuates, but no parasites have been identified in the stool. He has a severe arthropathy, with effusions, and progressive flexion contractures of both elbows. This patient has been reported previously in the context of his salmonellosis. ${ }^{6}$

Case 2

Correspondence: G. Spickett, D.Phil., M.R.C.P.

Accepted: 15 October 1990

A male, born in 1965, first presented aged 2 with a 2 month history of chronic diarrhoea, for which no 
cause was found and which resolved spontaneously. From 1971 to 1974 , he had recurrent sinopulmonary infections and otitis media, requiring antibiotics. In 1974, he had a tonsillectomy, adenoidectomy and bilateral myringotomies. In 1975 he developed intractable bilateral antral sinusitis, requiring repeated washouts, which was followed by lobar pneumonia. Chest radiographs following this were suggestive of bronchiectasis and CVID was diagnosed in 1976. In 1978 weekly intramuscular immunoglobulin injections were commenced at $25 \mathrm{mg} / \mathrm{kg}$ body weight: this led to a slight reduction in the frequency of sinopulmonary infections. In 1988 he had septic arthritis of the knee, and was then started on IVIg therapy, $16 \mathrm{~g}$ fortnightly, with a marked reduction in frequency and severity of sinopulmonary infections. Vitiligo was first noted in 1970 . One brother also has vitiligo, and his maternal grandfather may also have it. In 1982 he developed alopecia areata, which recovered spontaneously. This recurred in 1987, and did not respond to topical steroids. By 1988 , the alopecia was so widespread that he found shaving his head was more cosmetically acceptable, and since then there has been little sign of regrowth; other areas of the body are now affected.

\section{Case 3}

A man, born in 1932, was first diagnosed as hypogammaglobulinaemic in 1979, following a 12 year history of recurrent infections particularly of the respiratory tract and skin. There had been three recent admissions for pneumonia. When seen he was unwell with weight loss and a chronic productive cough and dyspnoea. A diagnosis of common variable hypogammaglobulinaemia was made and he was commenced on intramuscular immunoglobulin and fresh plasma infusions. There was no improvement in his health until he commenced on IVIg in 1984, receiving a dose of $15 \mathrm{~g}$ fortnightly. Alopecia areata was first noticed in 1979 and quickly progressed to alopecia totalis. He claimed to have suffered from two previous episodes of alopecia, in early childhood and late teens, both episodes recovering spontaneously. He also suffered from two severe episodes of eczema in 1980 and 1986, requiring treatment with systemic steroids.

\section{Case 4}

A man, born in 1953, was found to have CVID in 1981 following four episodes of pneumonia during the previous year. He had a chronic productive cough and investigation showed bilateral bronchiectasis. He had also had problems with recurrent sinus infections, necessitating sinus washouts in 1979. He commenced on replacement therapy with intramuscular immunoglobulin but changed to IVIg in 1986 following recurrent bronchitis. Widespread vitiligo was first noted in 1984 , and had appeared over a period of several months. $\frac{\varrho}{c}$ Though his health has remained good on a small dose (5 g monthly) of IVIg, his vitiligo has continued to spread slowly.

\section{Discussion}

Vitiligo is a relatively common condition affecting about $1 \%$ of the population. Severe alopecia is less. common. It has been suggested that vitiligo is an $\vec{\overrightarrow{ }}$ autoimmune mediated condition, based on its ${ }^{\omega}$ strong association with other organ specific autoimmune conditions such as pernicious 3 . anaemia, diabetes mellitus, Addison's disease andothyroid disorders..$^{7-9}$ In a minority of patients the $-v$ edges of the lesions have a dermal lymphocytico on infiltrate and there is a reduction in the number of $\mathrm{N}$ melanocytes. Circulating IgG antibodies against $\stackrel{\oplus}{-}$ melanocytes have been demonstrated in some응 patients with vitiligo. ${ }^{10,11}$ It is not clear whetherthese autoantibodies are pathogenic or merely an? epiphenomenon. Naughton has documented a correlation between the level of antibody and $\supset$ degree of depigmentation, and also claims that $\overrightarrow{0}$ these antibodies will lyse melanocytes by bo complement-mediated and antibody-dependext cellular cytotoxicity. ${ }^{12,13}$ Bursectomy, in an animal model of vitiligo, delays the development of depigmentation in DAM chickens prone to post-ฏ natal amelanosis, which may indicate that $B$ cells $\frac{0}{D}$ play a role in the depigmentation, although the melanocytes also have pre-existing biochemicalo defects. ${ }^{14}$ Studies in vit-vit mice have shown a loss of normal cutaneous immune responses. ${ }^{15}$ As always, it is difficult to extrapolate from animal models to human disease. Similar histological $\frac{0}{3}$ features are seen in alopecia, with a perifollicular:infiltrate of lymphocytes, and some patients have 3 . antibodies to endothelial cells of the capillary network of the hair bulb. ${ }^{16}$ It is thought that abnormalities of cell-mediated immunity may be involved in alopecia, although the evidence is not compelling. ${ }^{17}$ Hair growth may return spontan $-\frac{D}{0}$ eously or be induced by minoxidil. Initially this was thought to be due to local vasodilatory action, but $N$ minoxidil has also been shown to have immuno- $N$ modulatory functions ${ }^{18}$ Thus, there is only circumstantial evidence directly linking alopecia and ${ }_{\sigma}^{\omega}$ vitiligo to specific alterations in autoimmunity.

The incidence of autoimmunity in CVID is about $20 \%$, based on an extensive review of 103 patients seen at a clinic in New York. Six per cent had more ${ }_{-}^{+}$ than one autoimmune disorder, but haemato- $-\overline{0}$ logical abnormalities comprised the bulk of instances of autoimmunity; organ-specific autoim- $\frac{\rho}{\mathbb{Q}}$ 
Table I Clinical features

\begin{tabular}{|c|c|c|c|c|}
\hline & Case 1 & Case 2 & Case 3 & Case 4 \\
\hline Sex & male & male & male & male \\
\hline $\begin{array}{l}\text { Age at diagnosis } \\
\text { of CVID }\end{array}$ & 19 & 11 & 45 & 27 \\
\hline \multicolumn{5}{|c|}{$\begin{array}{l}\text { Immunoglobulins at } \\
\text { diagnosis }\end{array}$} \\
\hline $\operatorname{IgG}(\mathrm{g} / \mathrm{l})$ & 3.3 & 2.83 & $\mathrm{n} / \mathrm{d}$ & $\mathrm{n} / \mathrm{d}$ \\
\hline $\operatorname{IgA}(\mathrm{g} / \mathrm{l})$ & 0.03 & $\mathbf{n} / \mathbf{d}$ & $\mathrm{n} / \mathrm{d}$ & 0.35 \\
\hline $\operatorname{IgM}(\mathrm{g} / \mathrm{l})$ & 0.45 & 0.3 & $\mathrm{n} / \mathrm{d}$ & $<0.16$ \\
\hline $\operatorname{IgE}(\mathrm{IU} / \mathrm{l})$ & 120 & $<1$ & - & - \\
\hline \multicolumn{5}{|l|}{ Age at diagnosis } \\
\hline Alopecia & 24 & 17 & 45 & - \\
\hline Vitiligo & no & yes & no & yes \\
\hline
\end{tabular}

$\mathrm{n} / \mathrm{d}=$ none detectable.

munity occurred in only 4 cases and only one patient was documented to have alopecia, and none vitiligo. ${ }^{2}$ Morrell in 1986 found no evidence for an increased incidence of diabetes mellitus in the families of 25 CVID patients, ${ }^{19}$ but wider surveys are needed. B12 deficiency and gastric atrophy occur with increased frequency in CVID, but, unlike classical pernicious anaemia, autoantibodies to gastric parietal cells are absent, and the gastritis is more generalized, without antral sparing. ${ }^{1}$ It is interesting that case 1 was noted, at the time his B12 deficiency was identified, to have anti-intrinsic factor but not gastric parietal cell antibodies. He also suffered from type I diabetes mellitus, but islet cell antibodies were not sought at the time of diagnosis. The other three cases do not have any evidence of other autoimmune phenomena, either haematological or organ-specific.

The immunological defects in CVID are extensive. B lymphocyte function is significantly impaired and T cells, NK cells and antigen presenting cells are also abnormal. There is, however, considerable variation in the extent of the defects between patients and in vitro testing has delineated at least 4 distinct patterns. ${ }^{20}$ The occurrence of alopecia totalis in a patient with congenital agammaglobulinaemia (X-linked agammaglobulinaemia; XLA) and almost undetectable immunoglobulin levels argues that antibody is unlikely to be a significant factor in the generation of hair loss: ${ }^{3}$ $T$ cell function in XLA is essentially normal. Whether alopecia, vitiligo or other autoimmune problems may occur in CVID as a result of disordered $T$ lymphocyte function or abnormal antibody production by residual B cells is not known. The occurrence of both these conditions in patients with primary immunodeficiencies and otherwise healthy individuals indicates that, if immune mechanisms are involved in the pathogenesis, then they are unaffected by the immune defect giving rise to the antibody deficiency. There are no available data suggesting that alopecia or vitiligo are more common in immunodeficient patients compared to the general population, which suggests that the immunological abnormalities of CVID do not predispose to vitiligo or alopecia. Vitiligo has been reported in association with HIV-related disease, particularly following multiple viral infections, ${ }^{21}$ and the occurrence of alopecia and vitiligo in CVID may be through infection rather than the primary immune defect, even though the infections that these patients suffer are mainly bacterial. All the patients that we report here have had major infective problems.

Previous reports have speculated that the alopecia might result from adverse reactions to intramuscular therapy. ${ }^{3,4}$ This is highly unlikely, as none of the patients described here have had any significant adverse reactions to immunoglobulin therapy. There is no evidence from these 4 cases that aggressive treatment with intravenous immunoglobulin has any effect on the alopecia or vitiligo.

\section{References}

1. Asherson, G.L. \& Webster, A.D.B. Diagnosis and Management of Immunodeficiency Disorders. Blackwell Scientific Publications, Oxford, 1980.

2. Cunningham-Rundles, C. Clinical and immunologic analyses of 103 patients with common variable immunodeficiency. $J$ Clin Immunol 1989, 9: 22-33.

3. Ipp, M.M. \& Gelfand, E.W. Antibody deficiency and alopecia. J Pediatr 1976, 89: 728-731.

4. Garcia, M.C., Fonseca, E., Pascual, D. et al. Hipogammaglobulinemia primaria y alopecia areata. Med Clin (Barc) 1983, 80: 717-719. 
5. Tan, R.S.H. \& Samman, P.D., Thymoma, acquired hypogammaglobinaemia, lichen planus and alopecia areata. Proc R Soc Med 1974, 67: 196-198.

6. Leen, C.L.S., Birch, A.D.J., Brettle, R.P., Welsby, P.D. \& Yap, P.L. Salmonella in patients with primary hypogammaglobulinaemia. J Infect 1986, 12: 241-245.

7. McGregor, B.C., Karz, H.I. \& Doe, R.P. Vitiligo and multiple glandular insufficiencies. JAMA 1972, 219: 724-725.

8. Bor, S., Feiwel, M. \& Chanarin, I. Vitiligo and its aetiological relationship to organ-specific autoimmune disease. $\mathrm{Br} J$ Dermatol 1969, 81: 83-88.

9. Korkij, W., Soltani, K., Simjee, S., Marcincin, P.G. \& Chuang, T.-Y. Tissue-specific autoantibodies and autoimmune disorders in vitiligo and alopecia areata: a retrospective study. J Cutan Pathol 1984, 11: 522-530.

10. Hertz, K.C., Gazze, L.A., Kirkpatrick, C.H. \& Katz, S.I. Autoimmune vitiligo. Detection of antibodies to melaninproducing cells. $N$ Engl J Med 1977, 297: 634-637.

11. Naughton, G.K., Eisinger, M. \& Bystryn, J.-C. Antibodies to normal human melanocytes in vitiligo. $J$ Exp Med 1983, 158: 246-251.

12. Naughton, G.K., Reggiardo, D. \& Bystryn, J.-C. Correlation between vitiligo antibodies and extent of depigmentation in vitiligo. J Am Acad Dermatol 1986, 15: 978-981.

13. Norris, D.A., Kissinger, R.M., Naughton, G.K. \& Bystryn, J.-C. Evidence for immunologic mechanisms in human vitiligo: patients' sera induce damage to human melanocytes in vitro by complement-mediated damage and antibodydependent cellular cytotoxicity. J Invest Dermatol 1988, 90: 783-789.
14. Lamont, S.J. \& Smyth, J.R. Effect of bursectomy on develop-气̨ ment of a spontaneous postnatal amelanosis. Clin Immunol Immunopathol 1981, 21: 407-411.

15. Amornsiripanitch, S., Barnes, L.M., Nordlund, J.J., Trinkle, L.S. \& Rheins, L.A. Immune studies in the depigmenting C57BL/Ler-vit/vit mice. An apparent loss of contact $\overline{\vec{N}}$ hypersensitivity. J Immunol 1988, 140: 3438-3445.

16. Nunzi, E., Hamerlinck, F. \& Cormane, R.H. Immunopathological studies on alopecia areata. Arch Der-흠 matol Res 1980, 269: 1-11.

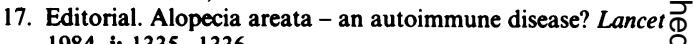
1984 , i: $1335-1336$

18. Fiedler-Weiss, V.C. \& Buys, C.M. Response to minoxidil in ڤొ severe alopecia areata correlates with $\mathrm{T}$ lymphocyte stimulation. Br J Dermatol 1987, 117: 759-763.

19. Morrell, D., Chase, C.L., Kupper, L.L. \& Swift, M. Diabetes mellitus in ataxia-telangiectasia, fanconi anemia, xeroderma $\vec{\omega}$ pigmentosum, common variable immune deficiency and $\overparen{ }$ severe combined immune deficiency families. Diabetes 1986,0 35: $143-147$.

20. Spickett, G.P., Webster, A.D.B. \& Farrant, J. Cellular abnormalities in common variable immunodeficiency. Immunodef Rev 1990, 2: 199-219.

21. Duvic, M., Rapini, R., Hoots, W.K. \& Mansell, P.W. Human o immunodeficiency virus-associated vitiligo: expression of $\mathrm{N}$ autoimmunity with immunodeficiency? J Am Acad Dermatol 1987, 17: 656-662. 